\title{
BMJ Open Self-harm and suicidal behaviour in Ghana: a systematic review protocol
}

\author{
Emmanuel Nii-Boye Quarshie (D), Kwaku Oppong Asante (D) , \\ Johnny Andoh-Arthur
}

To cite: Quarshie EN-B, Oppong Asante K, Andoh-Arthur J. Selfharm and suicidal behaviour in Ghana: a systematic review protocol. BMJ Open 2021;11:e053144. doi:10.1136/ bmjopen-2021-053144

- Prepublication history and additional supplemental material for this paper are available online. To view these files, please visit the journal online (http://dx.doi.org/10.1136/ bmjopen-2021-053144).

Received 06 May 2021 Accepted 12 November 2021

Check for updates

(c) Author(s) (or their employer(s)) 2021. Re-use permitted under CC BY-NC. No commercial re-use. See rights and permissions. Published by BMJ.

Department of Psychology, University of Ghana, Legon, Ghana

\section{Correspondence to}

Dr Emmanuel Nii-Boye Quarshie;

enquarshie@ug.edu.gh; enquarshie@gmail.com

\section{ABSTRACT}

Introduction Self-harm and suicidal behaviour represent major global health problems, which account for significant proportions of the disease burden in low-income and middle-income countries, including Ghana. This review aims to synthesise the available and accessible evidence on prevalence estimates, correlates, risk and protective factors, the commonly reported methods and reasons for self-harm and suicidal behaviour in Ghana.

Methods and analysis We will conduct a systematic review reported according to the Preferred Reporting Items for Systematic Reviews and Meta-Analyses statement (2009) recommendations. Regional and global electronic databases (African Journals OnLine, African Index Medicus, APA PsycINF0, Global Health, MEDLINE and PubMed) will be searched systematically up to December 2021 for observational studies and qualitative studies that have reported prevalence estimates, correlates, risk and protective factors, methods and reasons for self-harm and suicidal behaviour in Ghana. The electronic database searches will be supplemented with reference harvesting and grey literature searching in Google Scholar and ProQuest Dissertations \& Theses Global for postgraduate dissertations. Only records in English will be included. The Mixed Methods Appraisal Tool (2018) will be used to assess the methodological quality of included studies. Meta-analysis or narrative synthesis or both will be used, contingent on the extent of heterogeneity across eligible observational studies.

Ethics and dissemination Considering that this is a systematic review of accessible and available literature, we will not seek ethical approval. On completion, this review will be submitted to a peer-reviewed journal, be disseminated publicly at (mental) health conferences with focus on self-harm and suicide prevention. The important findings would also be shared with key national stakeholder groups in Ghana: Ghana Association for Suicide Prevention, Ghana Mental Health Authority, Ghana Psychological Association, Centre for Suicide and Violence Research, Accra and the Parliamentary Select Committee on Health.

Prospero registration number CRD42021234622.

\section{INTRODUCTION}

According to the WHO, self-harm ('intentional self-inflicted poisoning or injury, which may or may not have a fatal intent or outcome') ${ }^{1}$ and suicidal behaviour ('a range of behaviours that include thinking about

\section{Strengths and limitations of this study}

This is the first country-level systematic review of the prevalence, correlates, risk and protective factors, methods and reported reasons for self-harm and suicidal behaviour in Ghana.

- This protocol has been reported in accordance with the Preferred Reporting Items for Systematic Review and Meta-Analysis (PRISMA) Protocol guidelines.

- A senior information specialist will contribute to the development of the search strategies to ensure specificity, sensitivity and replication across all selected databases.

- On completion, the review will be reported according to the recommendations of the PRISMA-2009 statement.

- The criminal status of attempted suicide in Ghana and the lack of routinely collected data on (attempted) suicide in the country are likely to limit the key findings of this review.

suicide (or ideation), planning for suicide, attempting suicide and suicide itself' $)^{2}$ represent major global health problems that account for significant proportions of the global disease burden annually, particularly, in low and middle-income countries (LAMICs), including Ghana. ${ }^{3}$ In 2016, selfharm was ranked among the top 12 causes of death among persons aged 10-24 years in sub-Saharan Africa, ${ }^{4}$ and about $77 \%$ of global suicides in 2019 were recorded in LAMICs. ${ }^{5}$

Nonetheless, as observed in some LAMICs, ${ }^{6}$ attempted suicide is still a crime and remains highly stigmatised in Ghana, ${ }^{78}$ Section 57:1 of Ghana's Criminal Code provides that 'a person who attempts to commit suicide commits a misdemeanour, ${ }^{7}$ but there are growing efforts by various stakeholder groups and organisations in the country towards getting the Parliament of Ghana to consider repealing the antisuicide law. To date, only one national-level systematic review on 'mental health research in Ghana', published nearly a decade ago, is available, ${ }^{9}$ but without specific primary focus on self-harm or suicidal behaviour in the country. Also, two recent 
regional systematic reviews related to self-harm among young people ${ }^{10}$ and suicide in the general adult population ${ }^{11}$ have included data from Ghana. Even though these two regional reviews posit self-harm and suicidal behaviour as possible important public health challenges in Ghana, none of them provides a relatively whole picture of the phenomena within and across the general population of the country exclusively in one report. This is the gap in knowledge that the current review seeks to contribute evidence to address. Notably, emerging evidence suggests that Ghana is showing potential in the contribution of published literature on the phenomena of self-harm and suicidal behaviour. ${ }^{1011}$

Thus, it is timely to consider performing a systematic review that integrates and synthesises the evidence exclusively from Ghana on self-harm and suicidal behaviour. Potentially, the body of evidence from a carefully planned and performed country-level systematic review will, in part, better inform the ongoing (de)criminalisation debates and antistigma campaigns and provide a researchinformed basis for the development of contextually relevant and culturally sensitive intervention and prevention programmes in the country. Also, a country-level systematic review of the current nature can be informative in mapping out areas of self-harm and suicidal behaviour that require initial or expansive research attention.

Ghana is an Anglophone West African country with an estimated population of 30.9 million. ${ }^{12}$ The country is heterogeneous regarding ethnic groupings, language and religion. About $71.2 \%$ of Ghanaians identify as Christians, $17.6 \%$ as Muslims, $5.2 \%$ as African Traditional Religion adherents and $5.3 \%$ without any religious affiliation. ${ }^{13}$ The country is categorised as having a medium human development index (rank of 138), with a life expectancy of 64.1 years as of $2019 .{ }^{14}$ It is a lower middle-income country. ${ }^{15}$ In terms of the sustainable development goals, Ghana has an index score of $65.4 \%$, with a global ranking of 100 out of 166 countries. Ghana has the most comprehensive mental health legislation across Anglophone West Africa, ${ }^{16}{ }^{17}$ but there are still fundamental challenges with implementation, negative public attitudes and access. For example, the three psychiatric hospitals in the country are all located within two regions in the south-out of the 16 regions of the country; only $2.8 \%$ of persons requiring mental health services are able to access professional care. ${ }^{18}$ The most recent crude estimate by the WHO indicates that $6.6 / 100$ 000 population $($ women $=177$; men $=1816$ ) died by suicide in Ghana during 2019. ${ }^{5}$ The country is generally faced with an acute shortage of mental health professionals, and mental health facilities and services are significantly underfunded. ${ }^{18-20}$ In Ghana, the psychiatrist to population ratio is 0.058 per 100000 population, and 0.065 psychologists per 100000 population-which is less than enough compared with the WHO benchmark of one psychiatrist/100 000 population. ${ }^{21}$

Prior to designing and developing this review protocol, we searched key systematic review repositories and protocol registers, including the International Prospective Register of Systematic Reviews (PROSPERO), the Cochrane Library, Joanna Briggs Institute Database of Systematic Reviews and Implementation Reports and the Campbell Collaboration. We also searched peer-reviewed journals such as BMJOpen and BMC Systematic Reviews, and the key electronic databases selected for this review, to identify same or similarly worded-recently_published prospective or completed systematic reviews, specifically on self-harm and suicidal behaviour in Ghana. However, we found no such (prospective or completed) reviews; thus, no national-level systematic review exclusively focused on self-harm and suicidal behaviour in Ghana has been published.

\section{Objectives}

The objectives of this review will be to:

- Describe the reported prevalence estimates of selfharm and suicidal behaviour (ideation, planning and attempt) in Ghana.

- Describe the methods and means of self-harm and suicide attempt that have been reported in Ghana.

- Examine the reported motivations/reasons for selfharm in Ghana.

- Identify the known common correlates, risk and protective factors and associated key sociodemographic factors of self-harm and suicidal behaviour (ideation, planning and attempt) in Ghana.

\section{METHODS}

The development of this systematic review protocol is being reported in accordance with the Preferred Reporting Items for Systematic Review and Meta-Analysis (PRISMA) Protocol guidelines. ${ }^{22}$ Notably, on completion, the reporting of this systematic review will be guided by the community-agreed statement of the PRISMA-2009. ${ }^{23}$ The title and key information about the design and methods of this proposed systematic review have been registered on the PROSPERO database.

\section{Eligibility criteria}

Table 1 summarises the inclusion and exclusion criteria that will guide the selection of potentially eligible records.

\section{Outcomes}

Potentially eligible studies must have assessed or measured self-harm or suicidal behaviour (ideation, planning or attempt) as outcome in any age group in Ghana. Considering the criminal status of attempted suicide in Ghana and the lack of routinely collected official data on suicide in the country, we anticipate that eligible prevalence studies on suicidal deaths-where available-are likely to be based on medicolegal autopsy studies or police records, which are often less reflective of the true scope of the problem in the general population. ${ }^{24}$ Specifically, eligible studies must report evidence that addresses at least one of the objectives of this review: (a) reported period prevalence (lifetime, 12- month, 6-month or 1-month) estimates of self-harm and suicidal 
Table 1 Summary of inclusion and exclusion criteria

\begin{tabular}{|c|c|c|}
\hline Criterion & Include & Exclude \\
\hline Setting & $\begin{array}{l}\text { Primary studies conducted within non-clinical } \\
\text { contexts (ie, general population, community, } \\
\text { school-based, households/neighbourhoods, } \\
\text { homeless context, street-connected settings, } \\
\text { correctional facilities etc.) in Ghana. } \\
\text { Clinic-based studies conducted in Ghana } \\
\text { focused on self-harm or suicidal behaviour as the } \\
\text { main presenting condition. }\end{array}$ & $\begin{array}{l}\text { Studies focused on self-harm or suicidal } \\
\text { behaviour, but participants selected from a } \\
\text { geographical context outside Ghana. }\end{array}$ \\
\hline Participants & $\begin{array}{l}\text { Participants sampled from Ghana regardless } \\
\text { of age, gender, sexual or gender orientation, } \\
\text { occupational group, religious groupings, health } \\
\text { status, or 'alternative' subcultures (eg, cult } \\
\text { groups, Goth, Emo, Metallers, Punk). } \\
\text { Participants in eligible studies who have had } \\
\text { personal experiences of self-harm or suicidal } \\
\text { behaviour. }\end{array}$ & $\begin{array}{l}\text { Cross-national studies involving Ghana but with } \\
\text { study results not disaggregated by country. } \\
\text { Studies involving participants who report no } \\
\text { personal experience of self-harm or suicidal } \\
\text { behaviour. }\end{array}$ \\
\hline $\begin{array}{l}\text { Prevalence } \\
\text { estimate }\end{array}$ & $\begin{array}{l}\text { Studies must specify time frames within which } \\
\text { prevalence of self-harm and/or suicidal behaviour } \\
\text { was assessed. }\end{array}$ & $\begin{array}{l}\text { Time frame of prevalence estimates cannot be } \\
\text { determined, even after contacting authors. } \\
\text { Study reports with no clear indication of sample } \\
\text { size and population denominator. }\end{array}$ \\
\hline
\end{tabular}

behaviour in Ghana, (b) methods and means of self-harm and suicide in Ghana, (c) motivations/reasons for selfharm in Ghana and (d) correlates, risk and protective factors and key sociodemographic factors associated with self-harm and suicidal behaviour in Ghana. Potentially eligible studies estimating the prevalence of self-harm or suicidal behaviour must have specified the time frame or period of prevalence and clearly identified their method of assessment. Eligible prevalence studies will be considered for inclusion if they have a clear indication of the sample size and population denominator. Clinic-based (accident and emergency/outpatient/inpatient department) prevalence studies will be excluded due to the inherent bias in selection of patients and patients' access to clinical care that could skew results artificially. ${ }^{2526}$ For the same reason, studies reporting evidence of prevalence based on medicolegal autopsy studies or police records will be excluded. ${ }^{24} 27$

\section{Definition and measurement of outcomes}

Studies with clear definitions of self-harm or suicidal behaviour (ideation, planning and attempt) will be considered for inclusion. Although we anticipate eligible studies to distinguish between suicidal self-harm and non-suicidal self-harm in their findings, we are mindful to include all acts that meet the WHO definition of selfharm applied in this review-which does not require a specific intent. ${ }^{128}$ We will adopt this position because the literature is replete with debates about the clarity and usefulness (or otherwise) of the distinction between selfreported suicidal and non-suicidal acts of self-harm, ${ }^{29}$ which can be particularly problematic in a country like 
Ghana, where attempted suicide is a crime and where different languages may not clearly reflect the distinction. ${ }^{730}$ Furthermore, we will include all acts that meet the WHO definition of self-harm applied in this review, as all acts of self-harm (regardless of attribution and intention) are associated with increased risk of death by suicide. ${ }^{1230}$ Thus, we will include all studies where it is clear that selfharm (regardless of apparent intent), suicidal ideation, suicidal planning or attempted suicide was the question that participants were asked.

Additionally, specific measurement or means of case identification/assessment (including self-report measures or diagnostic instruments) should have been reported by potentially eligible studies:

- assessment using a standardised clinician rating based on medical documentation or clinical interview.

- Clinic-based diagnostic case ascertainment at admission or by inspecting medical records, clinical chart reviews or other.

- Assessment based on interviews that were constructed ad hoc for the study or interviews for which validation data are available.

- Assessment by a self-report single item.

- Assessment by a self-report multiitem questionnaire for which validation data or psychometric information are available or unavailable.

Prevalence studies that access data from family will be excluded: only self-reported estimates will be considered for inclusion.

\section{Types of study}

This review will consider for inclusion primary studies that have used observational study designs (ie, cross-sectional studies, case-control studies and cohort studies). ${ }^{31} 32$ Evidence on prevalence estimates and associated factors of self-harm and suicidal behaviour (ideation, planning and attempt) will be drawn mainly from the eligible observational studies. Primary studies using qualitative methods (eg, interviews, focus groups, psychological autopsy studies, media content analyses, retrospective analyses of clinical records) will be considered for inclusion to provide evidence, mainly, on the reported motivations/reasons and methods/means of self-harm and attempted suicide. Besides elaborating self-reported motivations/reasons for self-harm and suicidal behaviour, eligible qualitative studies may also shed additional light on the roles that specific correlates, risks and protective factors play in relation to self-harm and attempted suicide. A study will be excluded if the data had been reported (in a relatively sufficient detail, including larger sample size) by an earlier or recent study based on the same data set. Peer-reviewed publications based on postgraduate theses will be considered for inclusion, but the theses will be consulted for further methodological details.

\section{Participants}

This review will consider for inclusion studies that involve children, adolescents and adults, regardless of gender, sexual orientation, occupational group or religious groupings sampled from Ghana. Participants in eligible studies must have had personal experiences of self-harm or suicidal behaviour (ideation, planning and attempt).

\section{Study setting}

This review will include studies conducted in Ghana or within any of its 16 administrative geographical regions. Specifically, potentially eligible studies must have been conducted within clinical or non-clinical contexts, including schools, households, community, homeless contexts, street-connected settings, rural, periurban or urban settings.

\section{Information sources}

We will develop search strategies for eligible literature using medical subject headings (MeSH) and text words related to self-harm, suicidal behaviour and Ghana. We will search six electronic databases: two regional-specific databases (African Journals OnLine (AJOL) and African Index Medicus (AIM)) and four global databases (APA PsycINFO, Global Health, MEDLINE and PubMed). The electronic database search will be supplemented with grey literature (ie, postgraduate dissertations and preprints). We will search ProQuest Dissertations \& Theses Global, and Google Scholar for postgraduate dissertations. For preprints, we will search the Social Science Research Network, medRxiv, Research Square and Open Science Framework. Additionally, we would search the references of key papers to harvest or chase the citations of potentially eligible papers; we would use Social Science Citation Index, Science Citation Index and Google Scholar ('Cited by' function) to search relevant forward citations of key papers. Beyond the database searching, we will contact authors of at least two eligible papers, requesting for copies of relevant unpublished papers from their personal records to be screened for inclusion. We will use the 'Similar articles' feature in PubMed and the 'Related articles' function in Google Scholar to locate additional studies. All searches will be limited to studies reported in English and involving human subjects. English is the formal language of Ghana and scientific works from the country are published in English. The anticipated completion date of this review is March 2022. Thus, the searches will be limited to the year of inception of the selected databases, first, up to December 2020, then updated to December 2021, towards the end of the review.

\section{Search strategy and process}

Our specific literature search strategies will be created with the assistance of a senior information specialist with expertise in systematic review searching on health topics related to LAMICs. Our search strategy would include keywords, truncation, Boolean logic operators (AND, OR, NOT) and MeSH terms as relevant to and appropriate for each selected database (eg, (self-harm OR self-poisoning OR self-injur* OR attempted suicide OR suicid*) AND (Ghana OR Accra OR Kumasi OR 
Tamale)). The geographic search filter would include 'Ghana' and names of the 16 regions and capital cities; these would include both earlier and current names. After a prototype, APA PsycINFO search strategy is developed and finalised, it will be adapted to the subject headings and syntax of the other databases (AJOL, AIM, Global Health, MEDLINE and PubMed). The APA PsycINFO search strategy will be developed first with input from the review team and then peer reviewed by the senior information specialist providing technical advice for this review. Appendix 1 shows the draft APA PsycINFO search strategy. The reporting of our searches will be guided by the Statement for Reporting Literature Searches in Systematic Reviews (PRISMA-S). ${ }^{33}$ Three authors (EN$\mathrm{BQ}, \mathrm{KOA}$ and JA-A) will run the searches and one author (ENBQ) will compile the search results and eliminate duplicates.

\section{Study records}

\section{Data management}

EndNote (V.X9.3.3) will be used to collate, handle and manage the results of the database searches, to remove duplicates of records and to access the full text of potentially eligible studies. Notably, the selected databases will be searched individually, but the results will be combined before removing duplicates.

\section{Selection process}

Two reviewers (KOA and JA-A) will independently screen the titles and abstracts of the identified records within the lens of the prespecified eligibility criteria. We will then screen the full text of potentially eligible studies for inclusion, also guided by our prespecified eligibility criteria for the review. We will refer to published protocols of eligible studies and associated online supplemental materials or contact authors of eligible studies (that are not available online) through telephone or email correspondence for additional relevant information or missing information, where the authors are unable to reach consensus, the specific papers will be referred to a third reviewer (EN$\mathrm{BQ}$ ) for resolution.

\section{Data collection process and data items}

We will design an extraction form to record relevant information from eligible studies. The extraction form will include information about authors, year of publication, setting of study (clinic-based or non-clinical, etc), sampling and sample size, study design, outcome measurement, key findings and study quality score. Following the definitions and nomenclature of self-harm and suicidal behaviour guiding, this systematic review, the extraction of information on self-harm, will include self-poisoning and self-injury; and suicidal behaviour will include suicidal ideation, suicidal planning, suicidal attempt and suicide. Two reviewers (KOA and JA-A) will independently extract data from the included studies and the extractions will be referred to a third reviewer (ENBQ) for completeness and accuracy check. The review team will resolve discrepancies through consensus for accuracy check of included studies. We will also contact study authors of eligible studies for additional information and accuracy check-where necessary—to resolve uncertainties.

\section{Risk of bias in individual studies}

Two reviewers would independently use the Mixed Methods Appraisal Tool (MMAT-2018). ${ }^{34} 35$ The MMAT2018 is robust for assessing and describing the methodological quality of different kinds of study designs: qualitative research, randomised controlled trials, nonrandomised studies, quantitative descriptive studies and mixed method studies. ${ }^{34-36}$ The MMAT-2018 has three reviewer rating options: 'yes' (there is clear information related to the criterion), 'no' (there is no information related to the criterion), or 'can't tell' (reported information related to the criterion is unclear or inappropriate). Where reviewers are unclear (can't tell), authors will be contacted for clarification. No study will be excluded based on quality rating.

\section{Data synthesis}

Meta-analysis or narrative synthesis or both will be used, contingent on the extent of heterogeneity across the eligible observational studies (reporting prevalence and associations). The $\mathrm{I}^{2}$ statistic would be used to assess the extent of heterogeneity across the eligible prevalence and association studies $\left(\mathrm{I}^{2}\right.$ value of zero=no heterogeneity; $\leq 25 \%=$ low heterogeneity; $50 \%=$ moderate heterogeneity and $\geq 75 \%$ = substantial heterogeneity) ${ }^{37-39}$ If zero or low heterogeneity is obtained, the prevalence estimates would be pooled in meta-analysis based on random effects model using Jamovi programming software package (V.1.8.1). If necessary, sensitivity analysis will be performed to assess risk of bias-to identify possible sources of heterogeneity (in this case, the analysis will be limited to only included studies with low risk of bias). Where appropriate, we will conduct subgroup analysis-for example, based on gender/sex, design of study or age groupings-to ensure that comparable studies are only those that have similar effect measures.

However, where there is substantial heterogeneity across eligible studies, the three-step approach to narrative synthesis would be followed to synthesise the evidence drawn from the eligible studies ${ }^{40}$ : '(1) organising the description of the studies into logical categories, (2) analysing the findings within each of the categories and (3) synthesising the findings across all included studies'. Median values and associated IQRs would be used to present the prevalence estimates. Also, tables and graphical displays (including forest plots) would be used to support the narrative synthesis or qualitative summary, although meta-analysis would not be performed. ${ }^{40} 41$ Where the eligible observational studies (reporting prevalence and associations) are sufficient, we will consider including only studies that present data as OR and adjust for confounding factors (eg, age, sex, etc). However, considering that Ghana is now showing potential in 
contributing published data on self-harm and suicidal behaviour, we anticipate including all types of data. The exploratory nature of this review and the high possibility of accessing a limited number of studies will make the inclusion of all types of data useful. Again, the three-step approach to narrative synthesis would also be followed to synthesise the evidence drawn from the eligible qualitative studies (on reported reasons/motivations for and means of self-harm and suicidal behaviour).$^{40}$

\section{Patient and public involvement}

No patient involved.

\section{DISCUSSION}

When completed, this review will be the first to provide a holistic systematic account of the accessible and available evidence on the prevalence, correlates, risk and protective factors, methods and reported reasons for self-harm and suicidal behaviour across the general population of Ghana. More importantly, we anticipate that evidence of this review will, among other things, serve as a useful resource contributing to the evidence base informing the push for the repeal of the antisuicide law in Ghana.

However, when pooling included studies within a metaanalysis, we anticipate the possibility of substantial variation across records, which may create a challenge for meaningful synthesis. This could be due to the broad approach guiding this systematic review; but we believe that a broad approach affords much value, including subgroup analysis exploring the differences and similarities in terms of correlates, risk and protective factors of self-harm and suicidal behaviour.

The quality of the evidence reported by eligible studies will be assessed, which could be useful for designing future research. Potentially, the evidence of this review will help in mapping out which domains and (vulnerable) groups require self-harm and suicide research attention; for example, children aged 12 years and younger, patients presenting with non-communicable chronic medical conditions and persons in correctional facilities. Relatedly, the results of this review can inform targeted and universal intervention and prevention programmes in Ghana. Broadly, we anticipate that this review's findings will be relevant and of interest to persons in academia, researchers, the clinical community and health policymakers in Ghana.

\section{Amendments}

Even though no amendments to the review methods described in this protocol are anticipated, in the event of necessary protocol amendments, we will provide the date of each amendment. Also, we will describe the needed change(s) and the rationale in this section (Amendments). Changes will not be included in the protocol. All the reviewers will approve all amendment(s). Additionally, any corresponding amendment(s) will be made in the PROSPERO registration records of this protocol.
Contributors $\mathrm{EN}-\mathrm{BQ}, \mathrm{KOA}$ and JA-A conceptualised the study. All the authors developed the initial search strategy and ENBQ performed the preliminary literature search. EN-BQ, KOA and JA-A wrote the first draft of the manuscript. All the authors reviewed, edited, and approved the final version of the manuscript. EN-BQ serves as guarantor for the contents of this paper.

Funding The authors have not declared a specific grant for this research from any funding agency in the public, commercial or not-for-profit sectors.

Competing interests None declared.

Patient consent for publication Not applicable.

Provenance and peer review Not commissioned; externally peer reviewed.

Data availability statement Data sharing not applicable as no datasets were generated and/or analysed for this study. No data are available. This is a systematic review protocol, hence no data have been accessed or available as yet. Data sharing not applicable as no datasets generated and/or analysed for this study.

Supplemental material This content has been supplied by the author(s). It has not been vetted by BMJ Publishing Group Limited (BMJ) and may not have been peer-reviewed. Any opinions or recommendations discussed are solely those of the author(s) and are not endorsed by BMJ. BMJ disclaims all liability and responsibility arising from any reliance placed on the content. Where the content includes any translated material, BMJ does not warrant the accuracy and reliability of the translations (including but not limited to local regulations, clinical guidelines, terminology, drug names and drug dosages), and is not responsible for any error and/or omissions arising from translation and adaptation or otherwise.

Open access This is an open access article distributed in accordance with the Creative Commons Attribution Non Commercial (CC BY-NC 4.0) license, which permits others to distribute, remix, adapt, build upon this work non-commercially, and license their derivative works on different terms, provided the original work is properly cited, appropriate credit is given, any changes made indicated, and the use is non-commercial. See: http://creativecommons.org/licenses/by-nc/4.0/.

\section{ORCID iDs}

Emmanuel Nii-Boye Quarshie http://orcid.org/0000-0002-8720-2355

Kwaku Oppong Asante http://orcid.org/0000-0002-0206-3326

Johnny Andoh-Arthur http://orcid.org/0000-0002-7036-1835

\section{REFERENCES}

1 WHO. Mental health gap action programme (mhGAP) intervention guide for mental, neurological and substance use disorders in nonspecialized health settings. 2nd edn. Geneva, Switzerland: WHO, 2016.

2 WHO. Preventing suicide: a global imperative. Geneva: WHO, 2014.

3 WHO. Suicide: key facts, 2021. Available: https://www.who.int/newsroom/fact-sheets/detail/suicide [Accessed 25 Jun 2021].

4 Naghavi M. Global burden of disease self-harm Collaborators. global, regional, and national burden of suicide mortality 1990 to 2016: systematic analysis for the global burden of disease study 2016. BMJ 2019;364:194.

5 WHO. Suicide worldwide in 2019 - global health estimates. Geneva: World Health Organization, 2021.

6 Mishara BL, Weisstub DN. The legal status of suicide: a global review. Int J Law Psychiatry 2016;44:54-74.

7 Assembly Press. Criminal offences act of Ghana. act 29, 1960. Accra, Ghana, 1960.

8 Osafo J, Akotia CS, Andoh-Arthur J, et al. Attempted suicide in Ghana: motivation, stigma, and coping. Death Stud 2015;39:274-80.

9 Read UM, Doku VCK. Mental health research in Ghana: a literature review. Ghana Med J 2012;46:29-38.

10 Quarshie EN-B, Waterman MG, House AO. Self-harm with suicidal and non-suicidal intent in young people in sub-Saharan Africa: a systematic review. BMC Psychiatry 2020;20:1-26.

11 Mars B, Burrows $\mathrm{S}$, Hjelmeland $\mathrm{H}$, et al. Suicidal behaviour across the African continent: a review of the literature. BMC Public Health 2014;14:606.

12 Ghana Statistical Service. Projected population by region and sex, 2010, 2015-2020, 2021. Available: https://www.statsghana.gov.gh/ [Accessed 14 Mar 2021].

13 Ghana Statistical Service. Population \& housing census report. 2013. Accra, Ghana: Ghana Statistical Service (GSS), 2010.

14 UNDP. Human development report 2020: the next frontier: human development and the Anthropocene. New York: United Nations Development Programme (UNDP), 2020. 
15 The world bank. world bank country and lending groups, 2021. Country classification. Available: https://datahelpdesk.worldbank. org/knowledgebase/articles/906519-world-bank-country-andlending-groups [Accessed 10 Mar 2021].

16 Esan O, Abdumalik J, Eaton J, et al. Global mental health reforms: mental health care in Anglophone West Africa. Psychiatric Services 2014;65:1084-7.

17 Walker GH, Osei A. Mental health law in Ghana. BJPsych Int 2017;14:38-9.

18 Roberts M, Mogan C, Asare JB. An overview of Ghana's mental health system: results from an assessment using the world Health organization's assessment instrument for mental health systems (WHO-AIMS). Int J Ment Health Syst 2014;8:16.

19 Jack H, Canavan M, Ofori-Atta A, et al. Recruitment and retention of mental health workers in Ghana. PLoS One 2013;8:e57940.

20 Quarshie ENB, Annor F, Tagoe T. Psychologists in ghana: analysis of the standing register. New Voices in Psychology 2016;12:55-69.

21 WHO. Global health observatory data repository: human resources data by country, 2019. Available: https://apps.who.int/gho/data/ node.main.MHHR

22 Moher D, Shamseer L, Clarke M, et al. Preferred reporting items for systematic review and meta-analysis protocols (PRISMA-P) 2015 statement. Syst Rev 2015;4:1.

23 Moher D, Liberati A, Tetzlaff J, et al. Preferred reporting items for systematic reviews and meta-analyses: the PRISMA statement. PLoS Med 2009;6:e1000097.

24 Adinkrah M. Epidemiologic characteristics of suicidal behavior in contemporary Ghana. Crisis 2011;32:31-6.

25 Adomakoh CC. A preliminary report on attempted suicides seen in a general Hospital in Ghana. Ghana Medical Journal 1975;14:323-236.

26 Roberts MA, Nkum BC. Deliberate self-harm in Ghana. Ghana Medical Journal 1989;23:81-7.

27 Der EM, Dakwah IA, Derkyi-Kwarteng L, et al. Hanging as a method of suicide in Ghana: a 10 year autopsy study. Pathol Discov 2016;4:2-5.
28 Platt S, Bille-Brahe U, Kerkhof A, et al. Parasuicide in Europe: the WHO/EURO multicentre study on parasuicide. I. Introduction and preliminary analysis for 1989. Acta Psychiatr Scand 1992;85:97-104.

29 Kapur N, Cooper J, O'Connor RC, et al. Non-suicidal self-injury $\mathrm{V}$. attempted suicide: new diagnosis or false dichotomy? $\mathrm{Br} \mathrm{J}$ Psychiatry 2013;202:326-8.

30 WHO. Practice manual for establishing and maintaining surveillance systems for suicide attempts and self-harm. Geneva: WHO, 2016.

31 Grimes DA, Schulz KF. An overview of clinical research: the lay of the land. Lancet 2002;359:57-61.

32 Vandenbroucke JP, von Elm E, Altman DG, et al. Strengthening the reporting of observational studies in epidemiology (STROBE): explanation and elaboration. PLoS Med 2007;4:e297.

33 Rethlefsen ML, Kirtley S, Waffenschmidt S, et al. PRISMA-S: an extension to the PRISMA statement for reporting literature searches in systematic reviews. Syst Rev 2021;10:39.

34 Hong QN, Pluye P, Fàbregues S, et al. Vp26 a critical appraisal tool for systematic mixed studies reviews. Int J Technol Assess Health Care 2018;34:166-66.

35 Hong QN, Pluye P, bregues S F. Mixed methods appraisal tool (MMAT), version 2018. Canada: Canadian Intellectual Property Office, Industry Canada, 2018.

36 Pluye P, Hong QN. Combining the power of stories and the power of numbers: mixed methods research and mixed studies reviews. Annu Rev Public Health 2014;35:29-45.

37 Higgins JPT, Thompson SG. Quantifying heterogeneity in a metaanalysis. Stat Med 2002;21:1539-58.

38 Higgins JPT, Thompson SG, Deeks JJ, et al. Measuring inconsistency in meta-analyses. BMJ 2003;327:557-60.

39 Zlowodzki M, Poolman RW, Kerkhoffs GM, et al. How to interpret a meta-analysis and judge its value as a guide for clinical practice. Acta Orthop 2007;78:598-609.

40 Petticrew M, Roberts H. Systematic reviews in the social sciences: a practical guide. John Wiley \& Sons: Oxford, 2006.

41 loannidis JPA, Patsopoulos NA, Rothstein HR. Reasons or excuses for avoiding meta-analysis in forest plots. BMJ 2008;336:1413-5 